\title{
X-Ray Image Denoising with Directional Support Value Transform
}

\author{
Sheng Zheng*a, Emile A. Hendriks ${ }^{\mathrm{b}}$, Bangjun Lei ${ }^{\mathrm{a}}$, Wei Hao ${ }^{\mathrm{a}}$ \\ ${ }^{a}$ Institute of Intelligent Vision and Image Information, China Three Gorges University, Yichang \\ 443002, China; ${ }^{\mathrm{b}}$ Information and Communication Theory Group, Faculty of Electrical Engineering, \\ Mathematics and Computer Science, Delft University of Technology, 2628CD Delft, The \\ Netherlands
}

\begin{abstract}
Under the support vector machine framework, the support value analysis-based image fusion has been studied, where the salient features of the original images are represented by their support values. The support value transform (SVT)-based image fusion approach have demonstrated some advantages over the existing methods in multisource image fusion. In this paper, the directional support value transform (DSVT) is applied to the denoising of some standard images embedded in white noise and the X-ray images. This directional transform is not norm-preserving and, therefore, the variance of the noisy support values will depend on the scales. And then we use the hard-thresholding rule for estimating the unknown support values in different scales and the thresholding is scale-dependent. The peak signal noise ratio (PSNR) is used as an "objective" measure of performance, and our own visual capabilities are used to identify artifacts whose effects may not be well-quantified by the PSNR value. The experimental results demonstrate that simple thresholding of the support values in the proposed method is very competitive with techniques based on wavelets, including thresholding of decimated or undecimated wavelet transforms.
\end{abstract}

Keywords: Weighted least squares support vector machine, directional support value transform, image denoising

\section{INTRODUCTION}

Endovascular repair (EVAR) of an abdominal aortic aneurysm is a widely used therapeutic alternative to open repair. Analysis of X-ray image pairs from fluoroscopic roentgenographic stereophoto-grammetric analysis (FRSA), which is based on the combination of digital biplane fluoroscopy and roentgenographic stereophotogrammetric analysis, is one of the basic methods to measure three-dimensional motion of stent-graft markers with a very high accuracy and precision $[1,2]$. To effectively detect and extract the markers from these X-ray images, we apply image denoising technique on these images. Over the last decade, a wide range of wavelet-based tools and ideas have been studied. From the very simple ideas like thresholding of the orthogonal wavelet coefficients of the noisy data, followed by reconstruction, to the substantial improvements in perceptual quality obtained by translation invariant methods based on thresholding of an undecimated wavelet transform [3], all kinds of approaches have been proposed. More recently, dual tree wavelet denoising methods were developed in the context of image denoising [4]. Also, variations on the basic schemes have been experimented, which include modifications of thresholding functions, level-dependent thresholding, and so on. And also, the ridgelet and curvelet transforms - which have been proposed as alternatives to wavelet representation of image data is also introduced to the image denoising [5].

In recent years, within context of the statistical learning theory and the structural risk minimization, the support vector machine (SVM) has been developed [6]. SVM is a powerful tool for data classification and function estimation, and has been applied to a wide variety of domains such as pattern recognition, and function estimation, etc. Especially, the development of the least square SVM (LS-SVM) [7], which resulted in a set of linear equations instead of a quadratic programming problem, extended the application of SVM to on-line applications. The introduction of mapping technique extends further the application of SVM to image processing areas [8], including edge detection [9], interpolation [10], object detection [11], etc. One important point in the SVM is that the data with larger support values can most possibly become the support vectors in the sparse SVM, since the sparse process exploits the fact that the support values have a physical meaning in the sense that they reveal the relative importance of the data points for contributing to the SVM model [12]. Under the SVM framework, the support value analysis-based image fusion method is developed [13], where

*zsh@ctgu.edu.cn; phone 86717 6393975; fax 867176394492

MIPPR 2009: Medical Imaging, Parallel Processing of Images, and Optimization Techniques, edited by Jianguo Liu, Kunio Doi, Aaron Fenster, S. C. Chan, Proc. of SPIE Vol. 7497, 74971K (C) 2009 SPIE · CCC code: 0277-786X/09/\$18 - doi: 10.1117/12.832653

Proc. of SPIE Vol. 7497 74971K-1 
the salient features of the original images are represented by their support values. The support value transform (SVT)based image fusion method have demonstrated some advantages over the existing methods in multisource image fusion [13] and MS Pan-sharpening of remote sensing images [14]. To represent edges more efficiently, several approaches have been proposed including the steerable pyramids, curvelets [5], and directional wavelet transforms based on complex filter banks [15] etc. These transform methods separate edges with different orientations in each image, and can give superior results in the fused images comparing to the standard discrete wavelet transform [5]. Based on this consideration, the directional information is introduced to improve the performance of standard SVT by the use of the weighted mapping LS-SVM [16]. In this paper, we apply this digital transforms to the denoising of some standard images embedded in white noise and the X-ray images from the FRSA system.

The rest of this paper is organized as follows. Sec. 2 illustrates the directional support value analysis method. Sec. 3 presents the support value analysis-based image denoising technique. The experimental results are provided in Sec. 4 and the conclusions are drawn in Sec. 5.

\section{DIRECTIONAL SUPPORT VALUE ANALYSIS}

In order to incorporate the prior information into the estimation based upon the mapping LS-SVM solution, one can weight the error variables $e_{k}=\alpha_{k} / \gamma$ by weighting factors $v_{k}$ [17]. This leads to the optimization problem:

$$
\min _{\mathbf{w}^{\bullet}, b^{\bullet}, \mathrm{e}^{\bullet}} J\left(\mathbf{w}^{\bullet}, b^{\bullet}, \mathbf{e}^{\bullet}\right)=\frac{1}{2} \mathbf{w}^{\bullet T} \mathbf{w}^{\bullet}+\gamma \frac{1}{2} \sum_{k=1}^{N} v_{k} e_{k}^{2}
$$

subject to $y_{k}=\mathbf{w}^{\bullet T} \varphi\left(x^{\bullet} x_{k}\right)+b^{\bullet}+e_{k}^{\bullet}, k=1, \cdots, N$

From the conditions for optimality and elimination of $\mathbf{w}^{\bullet}, \mathbf{e}^{\bullet}$ one obtains the KKT system

$$
\left[\begin{array}{cc}
0 & 1^{\mathrm{T}} \\
1 & \mathbf{\Omega}^{*}+\mathbf{V}_{\gamma}
\end{array}\right]\left[\begin{array}{l}
b^{\bullet} \\
\boldsymbol{\alpha}^{\bullet}
\end{array}\right]=\left[\begin{array}{c}
0 \\
\mathbf{Y}
\end{array}\right]
$$

where the diagonal matrix $\mathbf{V}_{\gamma}$ is given by

$$
\mathbf{V}_{\gamma}=\operatorname{diag}\left\{\frac{1}{w_{1}}, \ldots, \frac{1}{\mathcal{v}_{N}}\right\}
$$

and the corresponding solution of the weighted mapping LS-SVM is finally given by [17]

$$
b^{\bullet}=\mathbf{B Y}, \quad \boldsymbol{\alpha}^{\bullet}=\mathbf{A}(\mathbf{Y}-\overrightarrow{1} b)=\mathbf{A}(\mathbf{I}-\overrightarrow{1} \mathbf{B}) \mathbf{Y}=\mathbf{O}^{\bullet} \mathbf{Y}
$$

with $\mathbf{A}_{\gamma}=\boldsymbol{\Omega}_{\gamma}^{-1}, \mathbf{B}_{\gamma}=1^{\mathrm{T}} \boldsymbol{\Omega}_{\gamma}^{-1} / 1^{\mathrm{T}} \boldsymbol{\Omega}_{\gamma}^{-1} 1$, and $\boldsymbol{\Omega}_{\gamma}=K\left(\mathbf{x}_{i}, \mathbf{x}_{j}\right)+\mathbf{V}_{\gamma}$.

The choice of the weights $v_{k}$ is determined based upon the error variables $e_{k}=\alpha_{k} / \gamma$ from the (unweighted) LS-SVM case. The weighted factor $v_{k}$ is some prior information in estimation of the unknown variables. Here the prior information is modeled in the form of a discrete diagonal matrix which associates a weight value $v_{k}$ to each image pixel in the neighborhood of pixel $\left(r_{0}, c_{0}\right)$. The weight of a pixel reflects the importance the pixel has in the estimation of the underlying surface with the mapping LS-SVM. In order to enhance the definition of edges underlying image surface along the direction of the local edge, the solution is to adapt the shape of the neighborhood by weighting differently the importance of each pixel. One kind of weight function is given by

$$
v(r, c)=1 / \exp \left(-\frac{r_{\theta}^{2}}{2 \sigma_{r}^{2}}-\frac{c_{\theta}^{2}}{2 \sigma_{c}^{2}}\right)
$$

with $r_{\theta}=c \cos \theta+r \sin \theta, c_{\theta}=r \cos \theta-c \sin \theta$.where $\theta$ is the local edge orientation, and $\sigma_{r} \gg \sigma_{c}$. The value of $\sigma_{c}$ is chosen at least three times smaller than the average inter-edge distance. 
Similar to the orthogonal wavelet transform, we set $\theta$ to be 0 and 90 degree, horizontal and vertical direction orientation, and then we can deduce the horizontal and vertical support value filters $f_{\mathrm{h}}$ and $f_{\mathrm{v}}$ from the Eq. (4). From this the original image $\mathrm{P}$ can be decomposed into subbands in horizontal, vertical and diagonal orientations in the following method

$$
\begin{array}{ll}
\mathrm{H}=\operatorname{imfilter}\left(\mathrm{P}, f_{\mathrm{h}}, \text { 'replicate' }^{\prime}\right) ; & \mathrm{L}=\mathrm{P}-\mathrm{H} ; \\
\mathrm{LH}=\operatorname{imfilter}\left(\mathrm{P}, f_{\mathrm{v}}, \text { 'replicate' }^{\prime}\right) ; & \mathrm{LL}=\mathrm{L}-\mathrm{LH} ; \\
\mathrm{HH}=\operatorname{imfilter}\left(\mathrm{P}, f_{\mathrm{v}}, \text { 'replicate' }^{\prime}\right) ; & \mathrm{HL}=\mathrm{H}-\mathrm{HH} ;
\end{array}
$$

Based on these directional sub-bands of image, the directional support value transform (DSVT) can be developed by filling zeros in the basic directional support value filters and obtaining a series of multi-scale support value filters. Similar to the standard SVT, the DSVT is a multi-resolution transform with frame elements indexed by scale and location parameters. For the DSVT, many parameters of the weighted mapping LS-SVM need to be defined and refined, such as kernel function $K$ and parameter $\gamma$ and the $\sigma_{r}$ and $\sigma_{c}$. These parameters for the different focuses image fusion can be optimized. The parameter $\sigma$ in RBF kernel is set to be 0.6 and $\gamma$ is set to be 1 , the size of the vector space is set to be $5 \times 5$ pixels, and the $\sigma_{r}$ and $\sigma_{c}$ is set to be 40 and 0.5 respectively. The optimal horizontal support value filter is finally given as follow [16].

$$
\left[\begin{array}{ccccc}
-0.0000 & -0.0000 & -0.0000 & -0.0000 & -0.0000 \\
-0.0077 & -0.0166 & -0.0350 & -0.0166 & -0.0077 \\
-0.0255 & -0.1680 & 0.5548 & -0.1680 & -0.0255 \\
-0.0077 & -0.0166 & -0.0350 & -0.0166 & -0.0077 \\
-0.0000 & -0.0000 & -0.0000 & -0.0000 & -0.0000
\end{array}\right]
$$

\section{IMAGE DENOISING}

We now apply our digital transforms for removing noise from image data. Suppose that one is given noisy data of the form

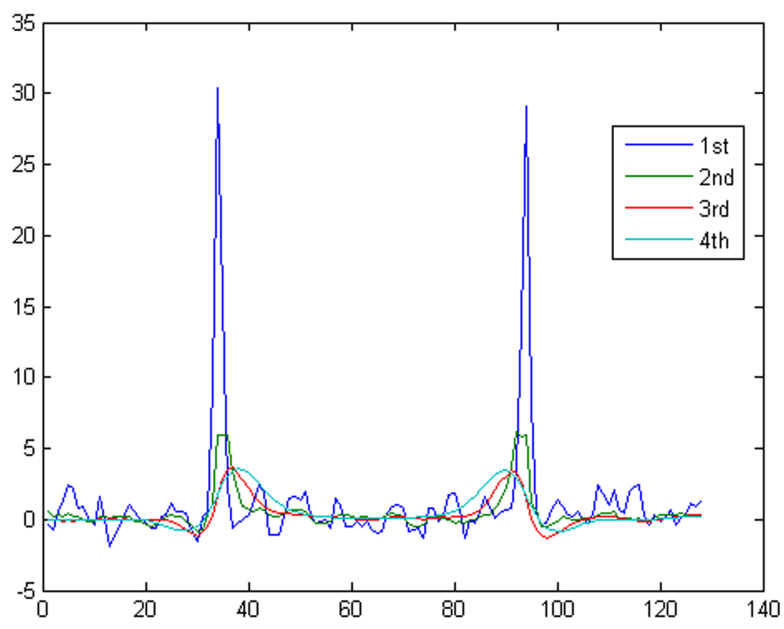

Figure 1 Profiles of support value images of synthetic image

$$
x_{i, j}=f(i, j)+\sigma z_{i, j}
$$

where $f$ is the image to be recovered and $z$ is white noise, i.e., $z_{i, j}^{i . i . d .} \sim N(0,1)$. Figure 1 shows the profiles of the support value images from the results of support value transform on a synthetic image. As shown in Figure 1, unlike FFTs or FWTs, the support value transform is not norm-preserving and, therefore, the variance of the noisy support values will depend on the support value index $\lambda$. For instance, letting $\boldsymbol{F}$ denote the discrete support value transform matrix, we have $\mathbf{F} z_{i, j}^{i . i . d .} \sim N\left(0, \mathbf{F} \mathbf{F}^{\mathrm{T}}\right)$. Because the computation of $\mathbf{F F}^{\mathrm{T}}$ is prohibitively expensive, we calculated an 
approximate value $\widetilde{\sigma}_{\lambda}^{2}$ of the individual variances using Monte-Carlo simulations where the diagonal elements of $\mathbf{F F}^{\mathrm{T}}$ are simply estimated by evaluating the support value transforms of a few standard white noise images.

Let $y_{\lambda}$ be the noisy support value coefficients $(y=\mathbf{F} x)$. We use the following hard-thresholding rule for estimating the unknown support values

$$
\begin{aligned}
& \hat{y}_{\lambda}=y_{\lambda}, \quad \text { if }\left|y_{\lambda}\right| / \sigma \geq k \widetilde{\sigma}_{\lambda} \\
& \hat{y}_{\lambda}=0, \quad \text { if }\left|y_{\lambda}\right| / \sigma \geq k \widetilde{\sigma}_{\lambda}
\end{aligned}
$$

In our experiments, we actually chose a scale-dependent value for $k$; we have $k=0.9$ for the first scale and $k=0.1$ for the second scale while $k=0.01$ for the others.

\section{IMAGE DENOISING EXPERIMENTS}

In our first example, a Gaussian noise with a standard deviation equal to 20 was added to the classical Lenna image (512 $\times 512$ ). Several methods were used to filter the noisy image.

1) Thresholding of the proposed directional support value transform (DSVT).

2) Hard thresholding of decimated wavelet transform.

3) Thresholding of curvelet transform [10].

5) Hard thresholding of dual tree wavelet transform, only the real part is used here.

We use the peak signal noise ratio (PSNR) as an objective measure of performance. In addition, we used our own visual capabilities to identify artifacts whose effects may not be well-quantified by the PSNR value. The sort of artifacts we are particularly concerned about may be seen on display in the upper right panel of Figure 2, which displays a wavelet reconstruction. This image has a number of problems near edges. In reconstructing some edges which should follow smooth curves one gets edges which are poorly defined and very choppy in reconstruction (for example in the crown of the hat); also some edges which are accurately reconstructed exhibit oscillatory structure along the edge which is not present in the underlying image (for example in the shoulder and the hat brim). We refer to all such effects as artifacts.

Our experiments are reported on Figure 2 and Figure 3. The latter figure represents a detail of the original image and helps the reader observe the qualitative differences between the different methods. We observe the following.

The support value reconstruction does not contain the quantity of disturbing artifacts along edges that one sees in wavelet reconstructions. An examination of the details of the restored images (Figure 3) is instructive. One notices that the decimated wavelet transform exhibits distortions of the boundaries and suffers substantial loss of important detail.

The curvelet transform enjoys superior performance over local ridgelet transforms, but it exhibits numerous small-scale embedded ridge-like blemishes.

The support value reconstructions display higher sensitivity than the wavelet-based reconstructions. But it exhibits numerous small-scale embedded point-like blemishes.

To study the dependency of the support value denoising procedure on the noise level, we generated a set of noisy images (the noise standard deviation varies from one to 100) from both Lenna and Barbara. We then compared the four different filtering procedures based, respectively, on the directional support value transform (DSVT), curvelet transform (Curvelet) and on the decimated (standard DWT) and dual tree complex (real DTWT) wavelet transforms. This series of experiments is summarized in Figure 4 which displays the PSNR versus the noise standard deviation. These experimental results show that the DSVT outperforms wavelets for removing noise from those images, as the DSVT PSNR is systematically higher than the wavelet PSNRs - and this, across a broad range of noise levels.

To measure three-dimensional motion of stent-graft markers with a very high accuracy and precision, we also apply the proposed method on the X-ray image obtained form the FRSA system. The Figure 5 shows the filtered results. As shown 
in Figure 5, the proposed support value reconstruction method provides more clear and more fidelity locations of the markers.
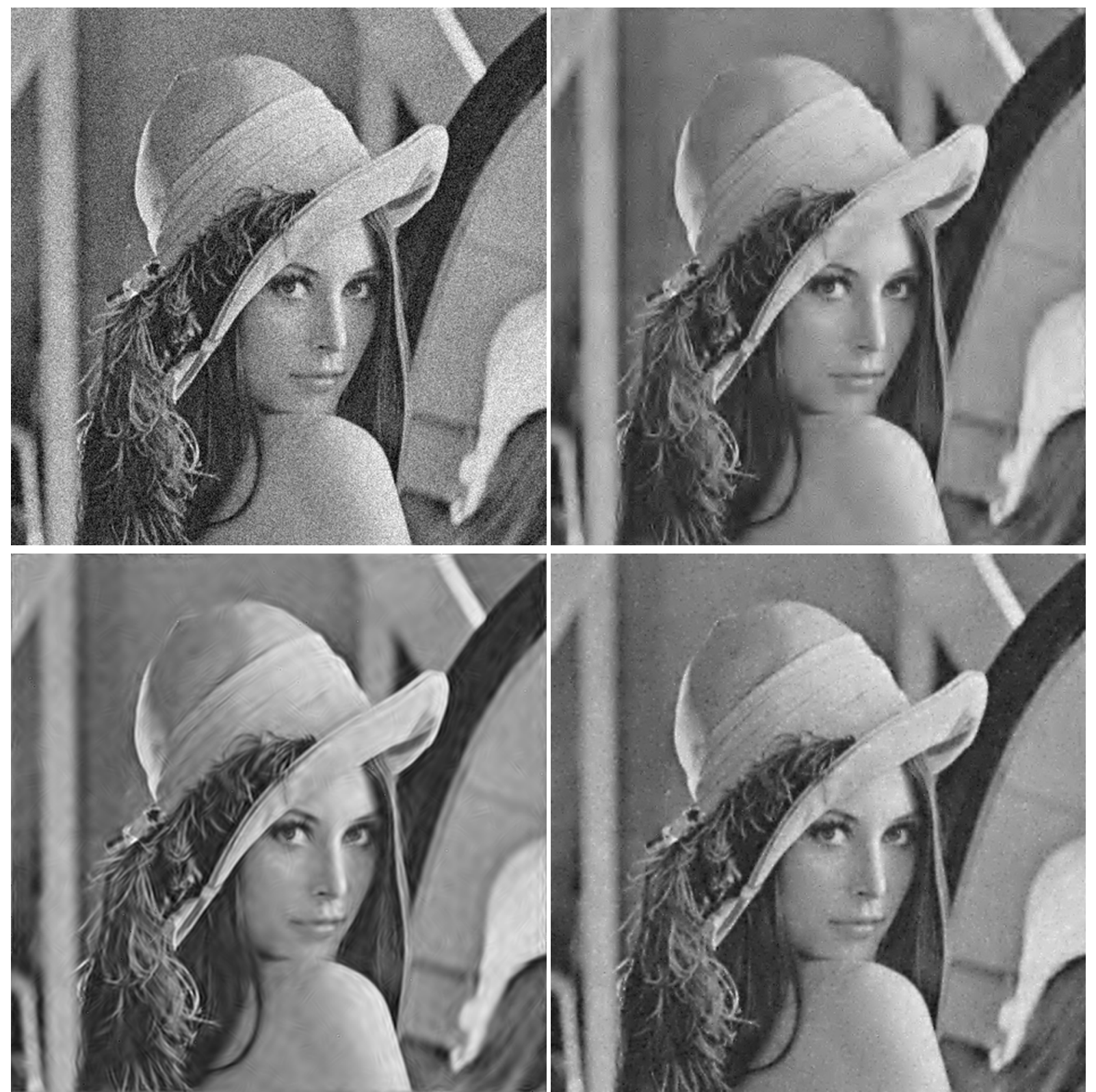

Figure 2 (Top left) Noisy image and (top right) filtered images using the decimated wavelet transform, (bottom left) the curvelet transform and the (bottom right) support value transform.

\section{CONCLUSIONS}

In this paper, we presented a strategy for digitally implementing the directional support value transform. And also apply the proposed transform on the image denoising. The work presented here is an initial attempt to address the problem of image denoising using digital analogs of some new mathematical transforms. Our experiments show that support value thresholding is encouraging, particularly as there seem to be numerous opportunities for further improvement. 

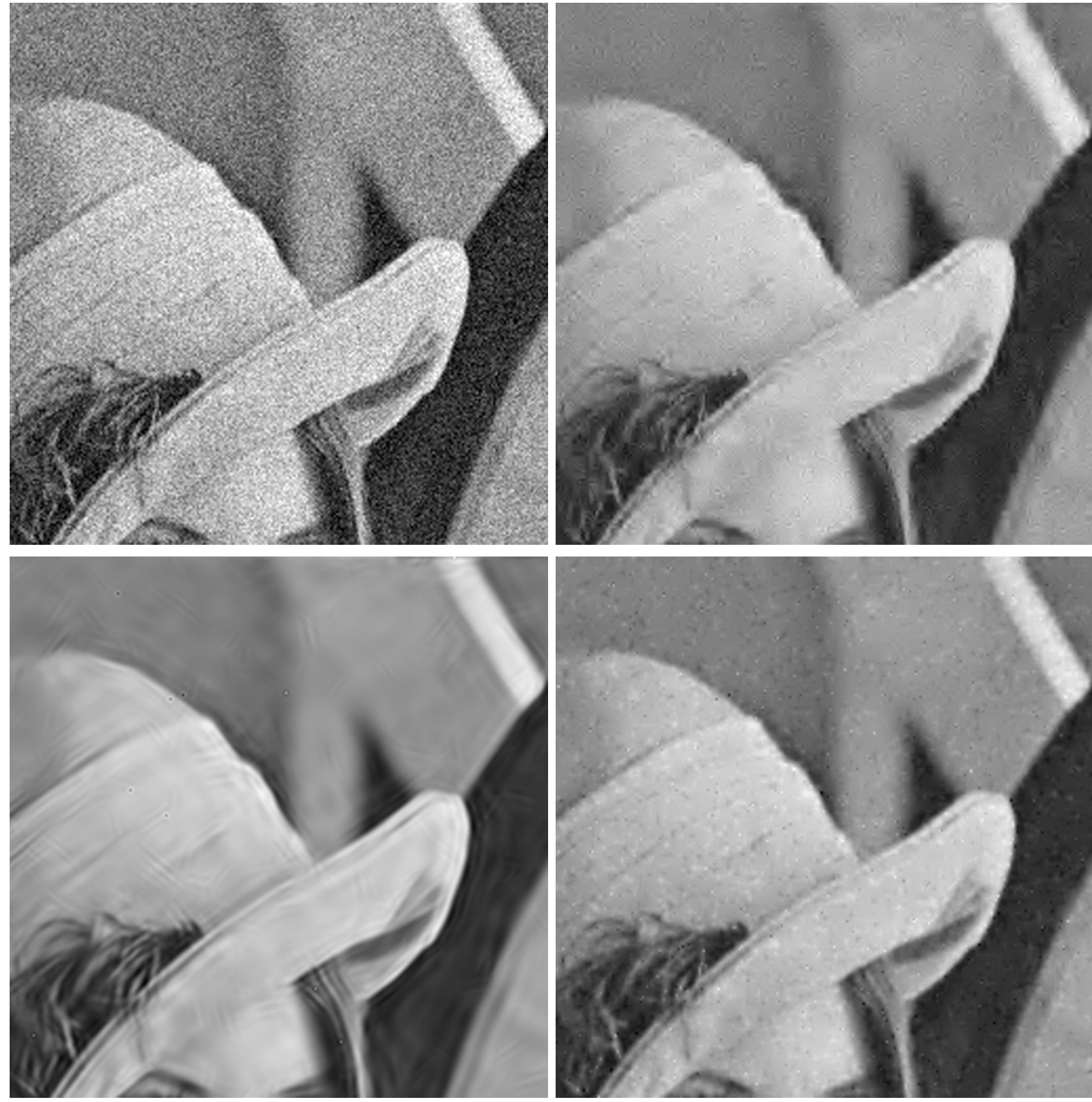

Figure 3 (Top left) Noisy image and (top right) filtered images using the decimated wavelet transform, (bottom left) the curvelet transform and the (bottom right) support value transform. The edges of the hat have been recovered with much greater fidelity in the support value transform approach.

\section{ACKNOWLEDGMENT}

This work was supported in part by funds from China National Natural Science Fund (Project No. 60875009), and the State Scholarship Fund awarded by China Scholarship Council. The authors would like to thank the Bart L. Kaptein and his colleagues for providing the X-ray image to test. 

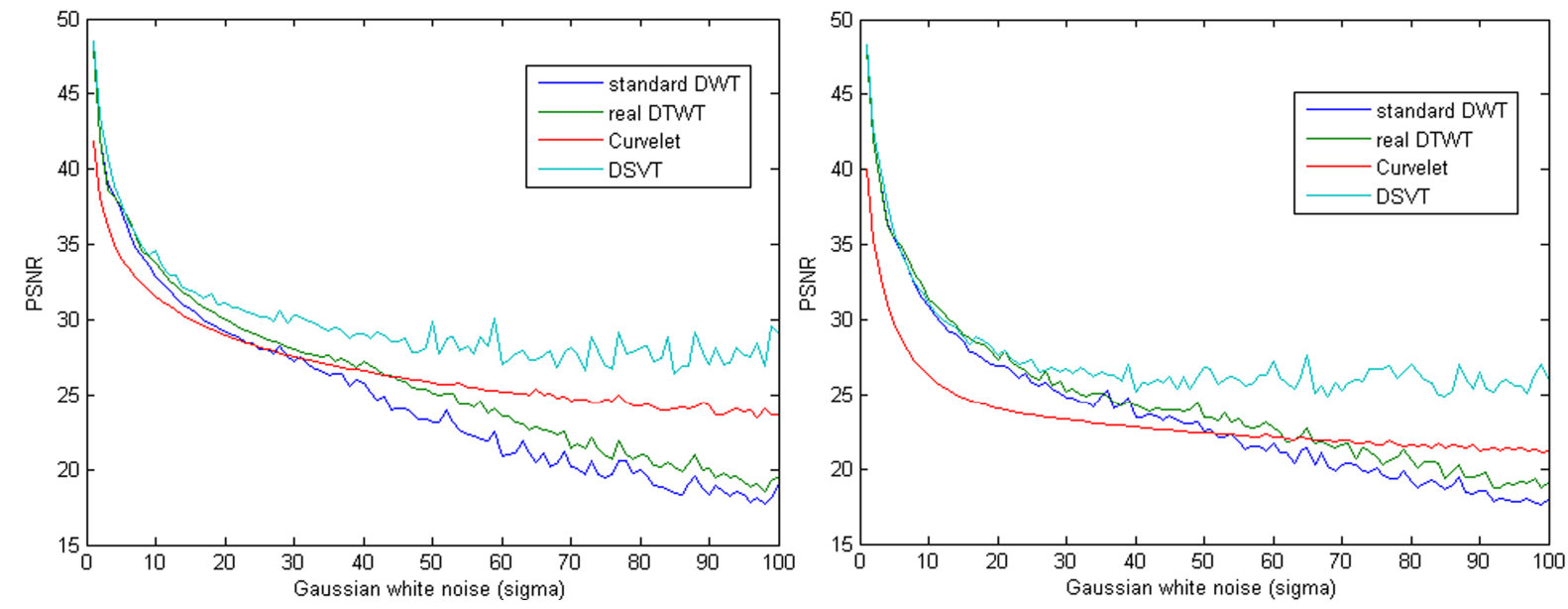

Figure 4 PSNR versus noise standard deviation for different denoising methods. The four methods based on the directional support value transform, curvelet, decimated and dual tree wavelet transforms are represented with different color lines, respectively. The left panel corresponds to Lenna, and the right to Barbara.

\section{REFERENCES}

[1] Koning OH, Garling EH, Hinnen JW, Kroft LJ, van der Linden E, Hamming JF, et al. "Accurate detection of stentgraft migration in a pulsatile aorta using roentgen stereophotogrammetric analysis", J Endovasc Ther, 14(1), 308 (2007).

[2] Koning OH, Kaptein BL, Garling EH, Hinnen JW, Hamming JF; Valstar ER; van Bockel J H, “Assessment of threedimensional stent-graft dynamics by using fluoroscopic roentgenographic stereophotogrammetric analysis", Journal of vascular surgery, 46(4), 773-9(2007).

[3] Starck JL, Fadili J, Murtagh F, “The undecimated wavelet decomposition and its reconstruction”, IEEE Transactions on Image Processing, 16(2), 297-309(2007).

[4] Kingsbury N, "Complex wavelets for shift invariant analysis and filtering of signals," Applied and Computational Harmonic Analysis, 10(3), 234-253(2001).

[5] Starck JL, Candes EJ, Donoho DL, "The curvelet transform for image denoising," IEEE Transactions on Image Processing, 11(6), 670-684(2002).

[6] Cortes C, Vapnik V, "Support vector networks", Machine Learning, 20, 273-297(1995).

[7] Suykens JAK, Vandewalle J, "Least squares support vector machine classifiers", Neural Processing Letters, 9(3), 293-300(1999).

[8] Zheng S, Sun YQ, Tian JW, Liu J, "Mapped least squares support vector regression”, International Journal of Pattern Recognition and Artificial Intelligence, 19(3), 459-475(2005).

[9] Zheng S, Liu J, Tian JW, “A new efficient SVM-based edge detection method”, Pattern Recognition Letters, 25(10), 1143-1154(2004).

[10] Zheng S, Tian JW, Liu J, Xiong CY, "Novel algorithm for image interpolation”, Optical Engineering, 43(4), 856865(2004).

[11] Zheng S, Liu J, Tian JW, "An efficient star acquisition method based on SVM with mixtures of kernels", Pattern Recognition Letters, 26(2), 147-165(2005).

[12] Suykens JAK, De Brabanter J, Lukas L, Vandewalle J, "Weighted least squares support vector machines: robustness and sparse approximation", Neurocomputing, 48, 85-105(1999).

[13] Zheng S, Shi WZ, Liu J, Tian JW, Zhu GX, "A multi source image fusion method using support value transform", IEEE Transaction on Image Processing, 16(7), 1831-1839(2007).

[14] Zheng S, Shi WZ, Liu J, Tian JW, "Remote sensing image fusion using multi-scale mapped LS-SVM", IEEE Transactions on Geoscience and Remote Sensing, 46(5), 1313-1322(2008). 
[15] Fernandes FCA, van Spaendonck RLC, Burrus CS, "A new framework for complex wavelet transforms", IEEE Transaction on Signal Processing, 51(7), 1825-1837(2003).

[16] Zheng S, Hendriks E.A., Lei B.J., Ye S.Z., "Different focuses image fusion with directional support value transform", International Conference on Machine Learning and Cybernetics, July 12-15, Baoding, China, (2009).

[17] Zheng S, Tian JW, Liu J, "Weighted MLS-SVM for approximation of directional derivatives", MIPPR 2005: Image Analysis Techniques, edited by Deren Li, Hongchao Ma, Proc. of SPIE Vol. 6044, 1-7(2005).
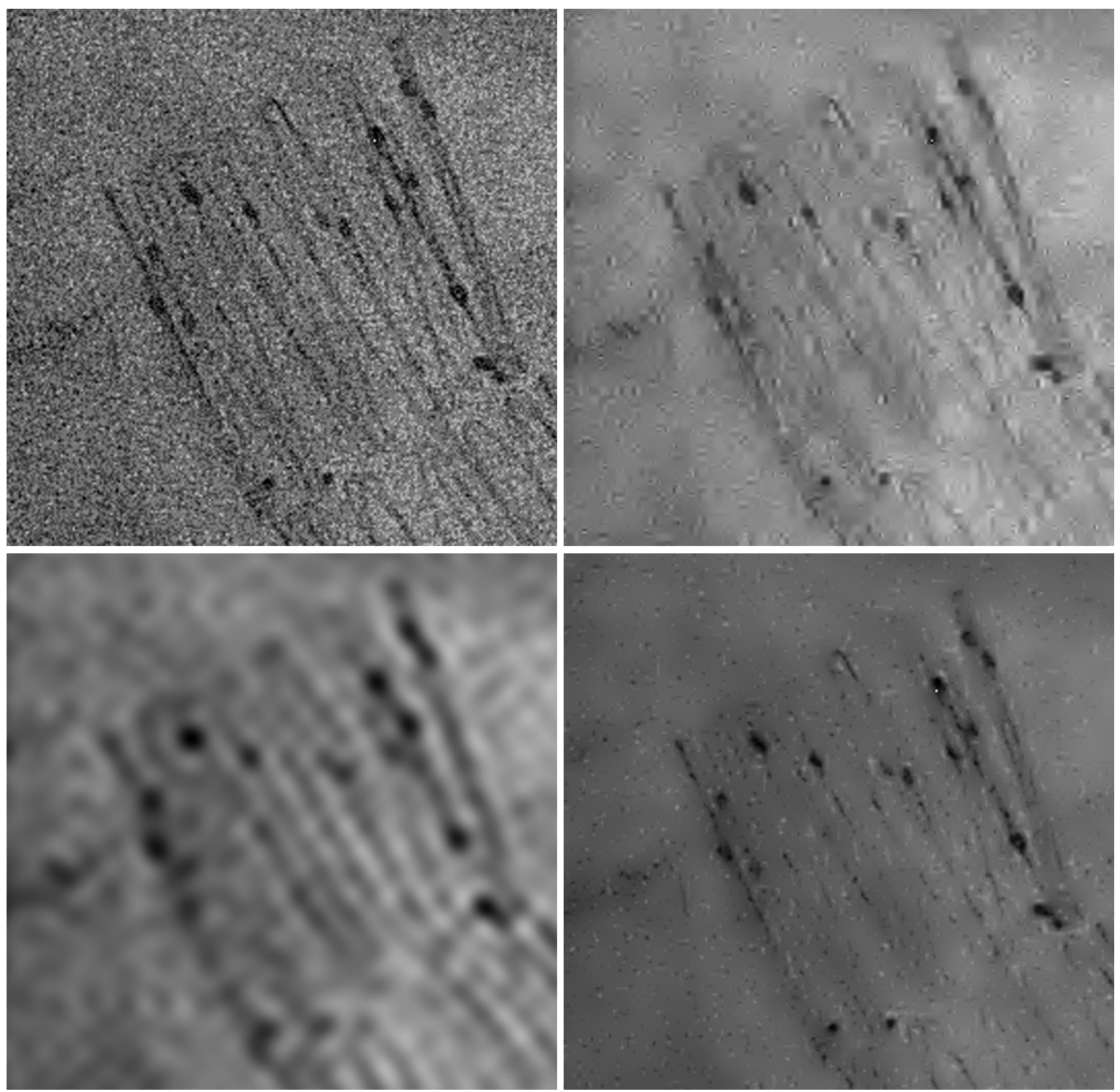

Figure 5 (Top left) X-ray image and (top right) filtered images using the decimated wavelet transform, (bottom left) the curvelet transform and the (bottom right) support value transform. 